\title{
Some results on boundary value problems for multivalued differential equations*
}

\author{
by Giuseppe Conti (Firenze) and Rita Iannacci (Cosenza)
}

\begin{abstract}
In this paper we give some existence theorems for the multivalued boundary value problem$$
x^{\prime}-A(t) x \in F(t, x),
$$

$$
L x=r .
$$

To do this we reduce the existence of solutions for this problem to that of a fixed point for a multivalued map.

The main tools we will use is a spectral theory for multivalued maps for the convex case and a selection theorem for the non-convex one.

Finally we give an application of our results and we show that, in this example, we cannot apply the previous results of other authors.
\end{abstract}

1. Introduction. Consider the multivalued differential system

$$
x^{\prime}-A(t) x \in F(t, x)
$$

with the linear condition

$$
L x=r .
$$

In [7] A. Lasota and Z. Opial reduced the existence of a solution for problem (1), (2) to that of a fixed point for a suitably defined multivalued map $T$. Then they employed the Kakutani-Ky Fan fixed point theorem to prove, under suitably hypotheses, that problem (1), (2) has solutions.

Using the same fixed point theorem, M. Grandolfi [4] and, with a different technique, L. E. Miller [8] extended the results of [7].

In this paper we calculate the asymptotic spectrum for the multivalued map $T$ as introduced in [5]. Using a subjectivity result given in [5], the spectrum calculus allows us to carry out an existence theorem for problem (1), (2).

- Work performed under the auspices of the C.N.R. (Italy). 
The paper is divided as follows. In Section 2 we collect all the notations and definitions to be used throughout the paper. Moreover, we state a spectrum property and some results which we need. In Section 3 we spend some words to recall the definition of the map $T$ and we calculate the spectrum of $T$.

In Section 4 we give an existence theorem for problem (1), (2). In Section 5 we apply our results to establish the existence of solutions for some multivalued boundary value problems and we give an example where we cannot apply the results of [4], [7], [8].

Finally in Section 6 we give an existence theorem for problem (1), (2) when $F(t, x)$ has not convex values.

2. Notations and definitions. Let $\Delta=[a, b]$ be a fixed compact interval of the real line $R$. Let $\boldsymbol{R}^{n}$ be the $n$-dimensional Euclidean space with Euclidean norm $\|\cdot\|$.

Let $C^{n}$ be the space of all continuous mappings from $\Delta$ into $R^{n}$ with the usual norm $\|x\|_{C}=\max _{t \in \Delta}\|x(t)\|$ and let $\left(L^{p}\right)^{n}(1 \leqslant p<\infty)$ be the Banach space of all $p$-summable mappings $f: \Delta \rightarrow R^{n}$ with the norm $\|f\|_{p}=\left(\int\|f(\tau)\|^{p} d \tau\right)^{1 / p}$. $\left(L^{\infty}\right)^{n}$ will denote the Banach space of essentially bounded maps $f: \Delta \rightarrow \boldsymbol{R}^{n}$ with the norm $\|f\|_{\infty}=\sup _{t \in A} \operatorname{ess}\|f(t)\| . L^{p}$ and $L^{\infty}$ will denote $\left(L^{p}\right)^{1}$ and $\left(L^{\infty}\right)^{1}$ respectively.

Given two real Banach spaces $E$ and $E^{\prime}$, a multivalued map with nonempty and compact values $F: E-\circ E^{\prime}$ is called upper (resp. lower) semicontinuous (briefly u.s.c. resp. l.s.c.) if the set $\{y: F(y) \cap A \neq \emptyset\}$ is closed (resp. open) in $E^{\prime}$ whenever $A$ is closed (resp. open) in $E$. If $F$ is l.s.c. and u.s.c., then $F$ is said to be continuous. Notice that a multivalued map $F$ with non-empty and compact values is u.s.c. if and only if its graph is closed on $E \times E^{\prime}$ and $F$ sends compact sets into relatively compact sets.

An u.s.c. map is said to be compact if it sends bounded sets into relatively compact sets.

A multivalued map $G: \Delta-O E$ with non-empty and closed values is called measurable if, for every $x \in E$, the distance from $x$ to $G(t)$ is a measurable function on $\Delta$.

$F: E-E^{\prime}$ is called quasibounded if it sends bounded sets into bounded sets and

$$
|F|=\limsup _{\|x\| \rightarrow+\infty} \frac{\delta(F(x))}{\|x\|}<+\infty,
$$

where $\delta(F(x))$ denotes $\sup _{y \in F(x)}\|y\|$.

The number $|F|$ is said to be the quasinorm of $F$. 
Let $F: E \multimap E^{\prime}$ be quasibounded. We put

$$
q(F)=\liminf _{\|x\| \rightarrow+\infty} \frac{\inf _{y \in F(x)}\|y\|}{\|x\|} .
$$

The spectrum of $F$ is the set defined by

$$
\Sigma(F)=\{\lambda \in R: q(\lambda I-F)=0\} \quad(\text { see }[5]),
$$

where $I$ is the identity on $E$.

In the sequel we shall use the following results: the first one, due to $R$. Iannacci [5] for the convex case and the second, due to M. Kisielewicz [6] for the non-convex case.

Proposition 2.1 ([5]). Let $E$ be a real Banach space and let $F: E \multimap E$ be convex-valued, compact and quasibounded. If $\lambda \neq 0$ belongs to either of the unbounded component of $\boldsymbol{R} \backslash \Sigma(F)$, then $\lambda I-F$ is onto.

Proposition 2.2 ([6]). Let $X$ be a separable Banach space and let $F: \Delta$ $\times X-O X$ be a multivalued map with non-empty and compact values. Assume that for every $x \in X, F(\cdot, x)$ is measurable; for every $t \in \Delta, F(t, \cdot)$ is continuous and there exists a function $m \in L^{1}$ such that $\delta(F(t, x)) \leqslant m(t)$ for $x \in X$ and a.e. $t \in \Delta$. Then there exists a continuous mapping $g: X \rightarrow L^{1}$ such that $g(x)(t) \in F(t, x)$ for each $x \in X$ and a.e. $t \in \Delta$.

3. The multivalued map $T$. Consider the multivalued differential system (1), (2) under the following hypotheses:

(a) $A: \Delta \rightarrow \mathscr{A}$, where $\mathscr{A}$ is the algebra of $n \times n$ matrices which are measurable and integrable on $\Delta$;

(b) $F: \Delta \times \boldsymbol{R}^{n} \rightarrow \boldsymbol{R}^{n}$ is a multivalued map such that:

$\left(b_{0}\right) F(t, x)$ is a non-empty, closed and convex subset of $\boldsymbol{R}^{n}$ for any $(t, x) \in \Delta \times R^{n}$,

$\left(b_{1}\right)$ for every fixed $x \in R^{n}, F(\cdot, x)$ is measurable on $\Delta$,

$\left(b_{2}\right)$ for every fixed $t \in \Delta$, the map $F(t, \cdot)$ is u.s.c. on $R^{n}$,

$\left(b_{3}\right)$ there are functions $\alpha, \beta \in L^{1}$ such that

$$
\sup _{y \in F(t, x)}\|y\| \leqslant \alpha(t)+\beta(t)\|x\|, \quad x \in \boldsymbol{R}^{n}, t \in \Delta \text { a.e.; }
$$

(c) $L$ is a linear continuous map from $C^{n}$ into $R^{m}$ and $r \in \operatorname{Im} L$ is fixed. An absolutely continuous map $x \in C^{n}$ will be called solution of (1) if it satisfies (1) almost everywhere on $\Delta$.

In what follows we shall need the definition of a multivalued map T. $C^{n} \multimap C^{n}$. This has been done already (see e.g. [1], [4] and [7]), but we will include it here for reader's convenience.

From condition (a) it follows that there exists only one function 
$U: \Delta \times \Delta \rightarrow \mathscr{A}$ which is continuous and such that

$$
U(t, s)=I+\int_{s}^{t} A(\tau) U(\tau, s) d \tau
$$

where $I$ is the identity on $\mathscr{A}$.

Also $U(\cdot, s)$ is a continuous and compact linear operator from $\boldsymbol{R}^{\boldsymbol{n}}$ into $C^{n}$, while the composition product $L_{U}=L \circ U(\cdot, s)$ is a linear operator from $\boldsymbol{R}^{n}$ into $\boldsymbol{R}^{\boldsymbol{m}}$, and hence can be represented by an $m \times n$ matrix.

Let $L_{U}^{*}$ be a generalized inverse of $L_{U}$, i.e., $L_{U} L_{U}^{*} L_{U}=L_{U}$. For a fixed $s \in \Delta$, let us define the linear operator $\Gamma:\left(L^{1}\right)^{n} \multimap C^{n}$ as follows

$$
\Gamma(f)(t)=-U(t, s) L_{U}^{*} L \int_{s}^{t} U(t, \tau) f(\tau) d \tau+\int_{s}^{t} U(t, \tau) f(\tau) d \tau .
$$

We recall that $\Gamma$ is continuous and compact also.

Let $c \in \boldsymbol{R}^{n}$ be a solution of $L_{U} c=0$. We put

$$
H r=U(t, s)\left(c+L_{U}^{*} r\right) .
$$

Let $\mathscr{F}(x)$ be the set of all measurable maps $y: \Delta \rightarrow \boldsymbol{R}^{n}$ such that $y(t) \in F(t, x(t))$ a.e. on $\Delta$. We recall that under assumption (b) on $F$, the map $\mathscr{F}: x \rightarrow \mathscr{F}(x)$ is bounded from $\left(L^{\infty}\right)^{n}$ into $\left(L^{1}\right)^{n}$ with non-empty, closed and convex values (see [7]).

For any $x \in C^{n}$ we set

$$
T(x)=\Gamma \mathscr{F}(x)+H r .
$$

The map $T: C^{n}-C^{n}$ is compact with non-empty, compact and convex values (see [7]).

Let us set

$$
\begin{aligned}
& M=\max _{t, s \in \Delta}\|U(t, s)\|, \\
& \alpha_{1}=\int_{\Delta} \alpha(\tau) d \tau, \\
& \beta_{1}=\int_{\Delta} \beta(\tau) d \tau .
\end{aligned}
$$

We have the following result:

THEOREM 3.1. The map $T$ defined above is quasibounded. If

$$
\exp \left(-M \beta_{1}\right)>M \| L_{U \|}^{*} \limsup _{\|x\|_{C} \rightarrow+\infty} \frac{1}{\|x\|_{C}}\left(\sup _{z \in \mathscr{F}(x)}\left\|L \int_{a}^{t} U(t, \tau) z(\tau) d \tau\right\|\right),
$$

then $\Sigma(T) \subset(-1,+1)$.

Proof. For any $x \in C^{n}$ we have $\delta(T(x)) \leqslant\|\Gamma\| \alpha_{1}+\|H r\|_{C}+\beta_{1}\|\Gamma\|\|x\|_{c}$. Dividing by $\|x\|_{c}$ and by taking the limsup as $\|x\|_{c} \rightarrow+\infty$, we obtain 
$|T| \leqslant\|\Gamma\| \beta_{1}$. Clearly $T$ sends bounded sets into bounded sets, hence $T$ is quasibounded.

Now we shall prove that if (iv) holds, then $\Sigma(T) \subset(-1,+1)$. Assume $|\lambda|$ $\geqslant 1$. Let $y \in \lambda x-\Gamma \mathscr{F}(x)-H r$. Hence there exists $z \in \mathscr{F}(x)$ such that $y=\lambda x$ $-\Gamma z-H r$.

For any $t \in \Delta$ we have $\|x(t)\| \leqslant|\lambda|\|x(t)\| \leqslant\|y\|_{c}+\|H r\|_{c}+\int_{a}^{t}\|U(t, \tau)\|\|z(\tau)\| d \tau+$

Using (i), for any $t \in \Delta$ we have

$$
+\|U(t, a)\|\left\|L_{U}^{*}\right\|\left\|L \int_{a}^{t} U(t, \tau) z(\tau) d \tau\right\|
$$

$$
\|x(t)\| \leqslant\|y\|_{C}+\|H r\|_{C}+M \int_{a}^{t}\|z(\tau)\| d \tau+M\left\|L_{U}^{*}\right\|\left\|L \int_{a}^{t} U(t, \tau) z(\tau) d \tau\right\| .
$$

Since $z(t) \in \mathscr{F}(t, x(t))$ a.e. on $\Delta$, assumption $\left(b_{3}\right)$ on $F$ implies that

$$
\|x(t)\| \leqslant\|y\|_{c}+\|H r\|_{c}+M \alpha_{1}+M \int_{a}^{t} \beta(\tau)\|x(\tau)\| d \tau+M\left\|L_{U}^{*}\right\|\left\|L \int_{a}^{t} U(t, \tau) z(\tau) d \tau\right\|
$$

By Gronwall's Lemma it follows that

$$
\|x(t)\| \leqslant\left(\|y\|_{c}+\|H r\|_{c}+M\left\|L_{v}^{*}\right\|\left\|L \int_{a}^{t} U(t, \tau) z(\tau) d \tau\right\|+M \alpha_{1}\right) \exp \left(M \beta_{1}\right)
$$

for any $t \in \Delta$.

By taking the sup for $t \in \Delta$, we obtain

$$
\exp \left(-M \beta_{1}\right)\|x\|_{C}-\left(\|H r\|_{C}+M \alpha_{1}+M\left\|L_{U}^{*}\right\|\left\|L \int_{a}^{t} U(t, \tau) z(\tau) d \tau\right\|\right) \leqslant\|y\|_{C}
$$

Therefore for any $\lambda$ with $|\lambda| \geqslant 1$ we get

$$
\begin{aligned}
& \inf _{y \in \lambda x-T(x)}\|y\|_{C} \\
& \geqslant \exp \left(-M \beta_{1}\right)\|x\|_{C}-\|H r\|_{C}-M \alpha_{1}-\sup _{x \in \mathscr{F}(x)} M\left\|L L_{U}^{*}\right\|\left\|L \int_{a}^{1} U(t, \tau) z(\tau) d \tau\right\| .
\end{aligned}
$$

We have readily

$$
q(\lambda I-T) \geqslant \exp \left(-M \beta_{1}\right)-M\left\|L_{u}^{*}\right\| \limsup _{\|x\| C \rightarrow+\infty} \frac{1}{\|x\|}\left(\sup _{z \in \mathcal{F}(x)}\left\|L \int_{a}^{t} U(t, \tau) z(\tau) d \tau\right\|\right) \text {. }
$$

From hypothesis (iv) it follows that $q(\lambda I-T)>0$ for $|\lambda| \geqslant 1$. Q.E.D.

Corollary 3.1. Assume that

$$
\exp \left(-M \beta_{1}\right)>M^{2}\left\|L_{v}^{*}\right\|\|L\| \beta_{1} .
$$

Then $\Sigma(T) \subset(-1,+1)$. 
Proof. We observe that for any $x \in C^{n}$ and for any $z \in \mathscr{F}(x)$, using assumption $\left(b_{3}\right)$ on $F$ we get

$$
\begin{aligned}
M\left\|L_{U}^{*}\right\|\left\|L \int_{a}^{1} U(t, \tau) z(\tau) d \tau\right\| & \leqslant M\left\|L_{U}^{*}\right\|\|L\| \int_{\Delta} M\|z(\tau)\| d \tau \\
& \leqslant M\left\|L_{U}^{*}\right\|\|L\|\left(M \alpha_{1}+M \beta_{1}\|x\|_{C}\right) .
\end{aligned}
$$

Now,

$$
\sup _{z \in \mathcal{F}(x)} M\left\|L_{U}^{*}\right\|\left\|L \int_{a}^{t} U(t, \tau) z(\tau) d \tau\right\| \leqslant M\left\|L_{U}^{*}\right\|\|L\|\left(M \alpha_{1}+M \beta_{1}\|x\|_{C}\right) .
$$

Thus

$$
\limsup _{\|x\|_{C} \rightarrow+\infty} \frac{1}{\|x\|_{C}}\left(\sup _{z \in \mathcal{F}(x)} M\left\|L_{U}^{*}\right\|\left\|L \int_{a}^{t} U(t, \tau) z(\tau) d \tau\right\|\right) \leqslant M^{2}\left\|L_{U}^{*}\right\|\|L\| \beta_{1} .
$$

Hence if (iv') holds, then (iv) also holds and Theorem 3.1 applies. Q.E.D.

4. An existence theorem. The previous spectral theorem allows us to obtain an existence theorem for the multivalued problem (1), (2).

We set $V_{r}=\left\{x \in C^{n}: L x=r\right\}$ and $\mathscr{L}_{r}=\left\{f \in\left(L^{1}\right)^{n}:\right.$ problem $\left(1^{\prime}\right): x^{\prime}$ $-A(t) x=f(t),(2): L x=r$ is solvable $\}$. Assume that (a) and (c) hold. From (c) it follows that $V_{r}$ is a closed linear variety of $C^{n}$. Note that either $\mathscr{L}_{r}=\varnothing$ or $\mathscr{L}_{r}$ is a closed linear variety of $\left(L^{1}\right)^{n}$.

We suppose that (c) and

$$
\mathscr{F}(v) \cap \mathscr{L}_{r} \neq \emptyset \quad \text { for every } v \in V_{r}
$$

hold.

Under these hypotheses, for any $v \in V_{r}$ we can consider the non-empty set defined by $T_{r}(v)=\Gamma\left(\mathscr{F}(v) \cap \mathscr{L}_{r}\right)+H r$.

LEMMA 4.1. If (a), (b), (c) and (H) hold, then the correspondence $x \rightarrow T_{r}(x)$ defines a compact multivalued map with convex values from $V_{r}$ into itself.

Proof. We observe that if $f \in \mathscr{L}_{r}$, then $\Gamma f+H r$ is a solution of problem (1'), (2) (see [4]). In particular we have that $T_{r}\left(V_{r}\right) \subset V_{r}$. Clearly for any $x \in V_{r}$, the set $T_{r}(x)$ is a non-empty and convex set.

Now we will prove that $T_{r}$ has closed graph. Clearly it is enough to show that $T_{r}-H r$ has closed graph.

Let $x \in V_{r}, z \in C^{n}$ and let $\left\{x_{n}\right\} \subset V_{r},\left\{z_{n}\right\} \subset C^{n}$ two sequences such that $z_{n} \in \Gamma\left(\mathscr{F}\left(x_{n}\right) \cap \mathscr{L}_{r}\right), n=1,2, \ldots$, and $\left\|x_{n}-x\right\|_{c} \rightarrow 0,\left\|z_{n}-z\right\|_{c} \rightarrow 0$.

For any $n=1,2, \ldots$ there exists $y_{n} \in \mathscr{F}\left(x_{n}\right) \cap \mathscr{L}_{r}$ such that $z_{n}=\Gamma y_{n}$.

Since $\left\|x_{n}-x\right\|_{c} \rightarrow 0$, we have that there exists a positive number $K$ (depending from $x$ ) such that $\left\|x_{n}\right\|_{c} \leqslant K, n=1,2, \ldots$ Hence from hypothesis $\left(b_{3}\right)$ it follows that $\left\|y_{n}(t)\right\| \leqslant \alpha(t)+K \beta(t)$, a.e. $t \in \Delta$ and $n=1,2, \ldots$

By Lemma 2 of [7] there exists a double sequence $\left\{\lambda_{n k}\right\}, n$ 
$=1,2, \ldots, k=n, n+1, \ldots$ of real non-negative numbers such that $\sum_{k=1}^{x} \lambda_{n k}=1, \quad \lambda_{n k}=0 \quad$ for sufficiently large $k$ (depending on $n$ ) and the sequence

$$
\tilde{y}_{n}=\sum_{k=n}^{\infty} \lambda_{n k} y_{k}, \quad n=1,2, \ldots,
$$

converges a.e. on $\Delta$ to a function $y \in\left(L^{1}\right)^{n}$.

With a standard technique (see Theorem 2 of [7]), we can show that $y(t) \in F(t, x(t))$ a.e. on $\Delta$, i.e. $y \in \mathscr{F}(x)$ and $\Gamma \dot{y}=z$.

On the other hand, since $\mathscr{L}_{\mathrm{r}}$ is a linear variety, by construction we have that $\tilde{y}_{n} \in \mathscr{L}_{r}, n=1,2, \ldots$ From the fact that $\mathscr{L}_{r}$ is closed, it follows that $y \in \mathscr{L}_{\text {r }}$ also. Therefore $z=\Gamma y \in \Gamma\left(\mathscr{F}(x) \cap \mathscr{L}_{r}\right)$.

Finally observe that, since $\Gamma$ is compact, then $T_{r}$ is compact also. Q.E.D.

We are now ready to prove an existence theorem for problem (1), (2).

THEOREM 4.1. Assume that hypotheses (a), (b), (c), (H), and (iv) (or (iv')) hold. Then problem (1), (2) has a solution.

Proof. We observe that a fixed point of the map $T_{r}: V_{r}-\circ V_{r}$ defined above is a solution of problem (1), (2). Then in order to prove our theorem it is enough to show that $T_{r}$ has a fixed point.

Since $r \in \operatorname{Im} L$, there exists $v_{0} \in C^{n}$ such that $L v_{0}=r$. Let us write $V_{r}$ in the form $V_{r}=v_{0}+W$, where $W$ denotes $\operatorname{Ker} L$. Since $L$ is continuous, $W$ and $V_{r}$ are closed. So that $W$ is a real Banach space with the norm induced by $C^{n}$.

For any $w \in W$ we set $S(w)=T_{r}\left(w+v_{0}\right)-v_{0}$. By Lemma 4.1 we have that the correspondence $w \multimap S(w)$ defines a compact multivalued map with convex values from $W$ into itself. Moreover, $S$ is quasibounded since $T_{r}(x) \subset T(x)$ for every $x \subset V_{r}$ and $T$ is quasibounded.

For any $\lambda \in R$ we have

$$
q(\lambda I-S)=\liminf _{\|w\|_{C} \rightarrow \infty} \frac{\inf _{y \in T_{r}\left(w+v_{0}\right)}\|\lambda w-y\|_{C}}{\|w\|_{C}} \geqslant q(\lambda I-T) .
$$

From (iv) (or (iv')) it follows that $q(\lambda I-T)>0$ for any $\lambda \in R$ with $|\lambda| \geqslant 1$.

Since $\Sigma(S) \subset \Sigma(T)$ and $\Sigma(T) \subset(-1,+1)$, by Proposition 2.1 we have that $I-S$ is onto. Therefore there exists $w_{0} \in W$ such that $w_{0} \in S\left(w_{0}\right)$, i.e., $w_{0}+v_{0} \in T_{r}\left(w_{0}+v_{0}\right)$. Now the proof is complete. Q.E.D.

As consequence of this theorem we obtain the following result due to $R$. Conti [2].

Corollary 4.1. Consider problem $\left(1^{\prime \prime}\right): \quad x^{\prime}-A(t) x=f(t, x), t \in \Delta$, (2): $L x=r$. 
Assume that

(I) $A \in \mathscr{A}$;

(II) $\|A(t)\| \leqslant \mu(t), t \in \Delta, \mu \in L^{1}$;

(III) $x(t) \equiv 0$ is the unique solution of the problem $x^{\prime}-A(t) x=0, L x=0$;

(IV) $f: \Delta \times \boldsymbol{R}^{n} \rightarrow \boldsymbol{R}^{n}$ is measurable in $t$ for every $x \in \boldsymbol{R}^{n}$ and continuous in $x$ for a.e. $t \in \Delta$;

(V) there exists $\varrho>0$ and a function $v_{Q}$ integrable on $\Delta$ such that $\|f(t, x)\| \leqslant v_{e}(t), t \in \Delta,\|x\| \leqslant \varrho$ and

$$
\varrho \geqslant\left\|U(t, a) L_{U}^{*} r\right\|_{C}+\left(1+\left\|U(t, a) L_{U}^{*} L\right\|\right) \exp \left(\int_{\Delta} \mu(\tau) d \tau\right) \int_{\Delta} v_{e}(\tau) d \tau
$$

Then problem (1"), (2) has a solution.

Proof. Let $\Delta^{\prime}=\{t \in \Delta: f(t, \cdot)$ is continuous $\}$. Define

$$
F(t, x)= \begin{cases}f(t, x) & \text { for } t \in \Delta^{\prime},\|x\| \leqslant \varrho, \\ f(t, x /\|x\|) & \text { for } t \in \Delta^{\prime},\|x\|>\varrho, \\ 0 & \text { for } t \in \Delta \backslash \Delta^{\prime}, x \in R^{n}\end{cases}
$$

Clearly $\|F(t, x)\| \leqslant v_{e}(t), t \in \Delta, x \in R^{n}$, hence hypothesis (iv') of Corollary 3.1 is satisfied.

Moreover, $V_{r}=C^{n}$, hence condition $(\mathrm{H})$ is also satisfied. Thus by Theorem 4.1 we have that problem $\left(1_{F}\right) x^{\prime}-A(t) x=F(t, x),(2) L x=r$ has a solution $x_{0} \in C^{n}$.

We claim that $\left\|x_{0}\right\|_{c} \leqslant \varrho$. Taking $c=0$ in the definition of $H r$ we have $\left\|x_{0}\right\|_{C} \leqslant\|\Gamma\|\left\|v_{\mathrm{\rho}}\right\|_{1}+\left\|U(t, a) L_{U}^{*} r\right\|_{c} \leqslant\|U(t, a)\|\left\|v_{\mathrm{Q}}\right\|_{1}+$

$$
\begin{aligned}
& +\|U(t, a)\|\left\|U(t, a) L_{U}^{*} L\right\|\left\|v_{\rho}\right\|_{1}+\left\|U(t, a) L_{U}^{*} r\right\|_{C} \\
& \leqslant\left(1+\left\|U(t, a) L_{U}^{*} L\right\|\right)\|U(t, a)\|\left\|v_{Q}\right\|_{1}+\left\|U(t, a) L_{U}^{*} r\right\|_{C} \\
& \leqslant\left(1+\left\|U(t, a) L_{U}^{*} L\right\|\right) \exp \left(\int_{\Delta} \mu(\tau) d \tau\right) \int_{\Delta} v_{Q}(\tau) d \tau+\left\|U(t, a) L_{U}^{*} r\right\|_{C} .
\end{aligned}
$$

Hence from (V) it follows that $\left\|x_{0}\right\|_{c} \leqslant \varrho$.

This implies that $F\left(t, x_{0}\right)=f\left(t, x_{0}\right)$, hence problem $\left(1^{\prime \prime}\right)$, (2) has a solution. Q.E.D.

5. Some remarks. In [8] L. E. Miller shoved the following theorem:

Theorem 5.1. Assume that conditions $(a),\left(b_{0}\right),\left(b_{1}\right),\left(b_{2}\right),(c)$ hold. Moreover, suppose that

$\left(\mathrm{b}_{3}^{\prime}\right)$ for all $\varrho>0$ there exists a function $H_{e} \in L^{1}$ such that $\sup \{\|y\|$ : $y \in F(t, x),\|x\| \leqslant \varrho\} \leqslant H_{Q}(t)$ for every $t \in \Delta$;




$\left(\tilde{H}_{1}\right) V_{r} \cap B_{a} \neq \phi$, where $B_{a}=\left\{x \in C^{n}:\|x\|_{c} \leqslant Q\right\}$,

$\left(\tilde{\mathbf{H}}_{2}\right) V_{r} \cap B_{0} \subset W_{r}=\left\{x \in C^{n}: \mathscr{F}(x) \cap \mathscr{L}_{r} \neq \phi\right\}$,

$\left(\tilde{\mathrm{H}}_{3}\right)\|\Gamma\| \int_{\Delta} H_{e}(\tau) d \tau+U(t, a) L_{U} r \leqslant \varrho$.

Then problem (1), (2) has a solution.

Proof. Observe that the closed and convex subset $V_{r} \cap B_{Q}$ is invariant under the map $T_{r}: V_{r}-\circ V_{r}$ defined in Section 4. Since $T_{r}$ is compact, the statement is an immediate consequence of the Kakutani-Ky Fan fixed point Theorem. Q.E.D.

Remark. Observe that in [8] $U(t, s)=\varphi(t) \varphi^{-1}(s)$, where $\varphi$ is the fundamental matrix for the linear homogeneous system $x^{\prime}=A(t) x$ with $\varphi(a)$ $=I$. However, Theorem 5.1 holds also in the general case.

The following results are an immediate consequence of Theorem 5.1.

Corollary 5.1 (M. Grandolfi [4]). Assume that conditions (a), (b), (c) hold. Moreover, assume that: (iv") $\|\Gamma\| \beta_{1}<1$ and

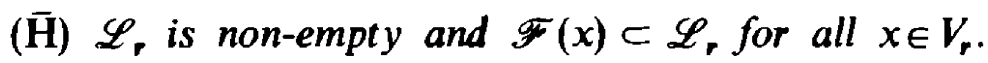

Then problem (1), (2) has solutions.

Corollary 5.2 (A. Lasota and Z. Opial [7]). Assume that (a), (b), (iv") hold. Moreover, assume that: $\left(\mathrm{c}^{\prime}\right) L$ is a continuous linear operator from $C^{n}$ into $\boldsymbol{R}^{n}, r \in \dot{R}^{n} ;(\boldsymbol{A}) x^{\prime}-A(t) x=0, L x=0$ has only the trivial solution.

Then problem (1), (2) has solutions.

In [4], [7] and [8] $\|\Gamma\|$ is not explicited, hence it seems that these results are not comparable with our results. However, we give now an application of our results; we will see that in this example, we cannot use the theorems of [4], [7] and [8].

5.1. Consider the following multivalued boundary value problem

$$
u^{\prime \prime}+u \in F\left(t, u, u^{\prime}\right), \quad 3 u(0)+u(\pi)=0, \quad 3 u^{\prime}(0)+u^{\prime}(\pi)=0
$$

We assume that $F:[0, \pi] \times \boldsymbol{R}^{2} \multimap \boldsymbol{R}$ is a multivalued map with non-empty, closed and convex values satisfying $\left(b_{1}\right) ;\left(b_{2}\right)$ and

$\left(b_{3}^{\prime}\right) \sup _{y \in F(t, v, w)}\|y\| \leqslant \alpha(t)+\beta(t)\left(v^{2}+w^{2}\right)^{1 / 2}, t \in[0, \pi], v, w \in R$, with $\alpha, \beta \in L^{1}$ and $\beta_{1}=\frac{2}{3}+\varepsilon, \varepsilon=\frac{2}{3}\left(2-\exp \frac{2}{3}\right) /\left(2+\exp \frac{2}{3}\right)$;

$\left(\mathrm{b}_{4}^{\prime}\right) F(0, v, w)=F(\pi, v, w), \forall v, w \in R$.

By standard calculations we can prove that $L_{U}=2 I$ and $L_{U}^{*}=\frac{1}{2} I$. Since $M=1$ and $\exp \left(-\beta_{1}\right)>\frac{1}{2} \beta_{1}$, it follows that hypothesis (iv) is satisfied and problem $(5.1)$ is solvable.

Take $y_{0}=\left(\begin{array}{l}\sin t \\ \cos t\end{array}\right)$; we have $\left\|\Gamma\left(y_{0}\right)(\pi)\right\|=\frac{3}{2} \pi$. Hence $\|\Gamma\|>\frac{3}{2}$ and $\|\Gamma\| \beta_{1}>1$. On the other hand $H_{\mathrm{p}}(t) \leqslant \alpha(t)+\beta(t) \varrho$, so that for any $\varrho>0$ we 
get $\|\Gamma\| \int_{\Delta} H_{Q}(\tau) d \tau=\|\Gamma\|\left(\alpha_{1}+\beta_{1} \varrho\right)>\|\Gamma\| \beta_{1} \varrho>\varrho$. Therefore we cannot apply Theorem 5.1.

6. The non-convex case. In this section we give an existence theorem for problem (1), (2) without the assumption that $F(t, x)$ is convex. However, we must assume that, for every $t \in \Delta, F(t, \cdot)$ is continuous on $R^{n}$. The main tool we will used is Proposition 2.2.

Theorem 6.1. Suppose that $(\mathrm{a}),\left(\mathrm{b}_{1}\right),\left(\mathrm{b}_{3}\right),(\mathrm{c})$, (iv') and $(\overrightarrow{\mathrm{H}})$ hold. Moreover, assume that, for every $(t, x) \in \Delta \times \boldsymbol{R}^{n}, F(t, x)$ is a non-empty and closed subset of $\boldsymbol{R}^{n}$ and, for every $t \in \Delta, F(t, \cdot)$ is continuous. Then problem (1), (2) has solutions.

Proof. Consider the map

$$
G(t, x)= \begin{cases}F(t, x) & \text { if }\|x\| \leqslant r, \\ F(t, r x /\|x\|) & \text { if }\|x\| \geqslant r,\end{cases}
$$

where

$$
0 \leqslant r \leqslant \frac{M^{2}\left\|L_{v}^{*}\right\|\|L\| \alpha_{1}+\|H r\|_{C}+M \alpha_{1}}{\exp \left(-M \beta_{1}\right)-M^{2}\left\|L_{v}^{*}\right\|\|L\| \beta_{1}}
$$

Clearly the map $G(\cdot, x)$ is measurable for every $x \in \boldsymbol{R}^{n}, G(t, \cdot)$ is continuous for every $t \in \Delta$ and $\delta(G(t, x)) \leqslant \alpha(t)+\beta(t) r$. Hence by Proposition 2.2 there exists a continuous map $g: \boldsymbol{R}^{n} \rightarrow L^{1}$ such that $g(x)(t) \in G(t, x)$ for each $x \in \boldsymbol{R}^{n}$, a.e. $t \in \Delta$.

Let us consider the singlevalued boundary value problem

$$
x^{\prime}-A(t) x=f(t, x)
$$

$$
L x=r
$$

where $f(t, x)=g(x)(t)$. It is easy to see that the hypotheses of our Theorem (see [3]) are fulfilled. Hence problem $\left(1_{f}\right),(2)$ has solutions.

In particular the multivalued problem

$$
x^{\prime}-A(t) x \in G(t, x)
$$

$$
L x=r
$$

has solutions. Since $g(t, x) \leqslant \alpha(t)+\beta(t)\|x\|$, using the Gronwall's Lemma and taking in account the hypothesis (iv'), it is easy to see that a solution $\bar{x}$ of problem $\left(1_{G}\right),(2)$ satisfies $\|\bar{x}\| \leqslant r$.

So that $\bar{x}$ is a solution of problem (1), (2) and the proof is complete. 


\section{References}

[1] S. R. Bernfeld, V. Lakshmikanthan, An Introduction to Nonlinear Boundary Value Problems, Academic Press, New York 1974.

[2] R. Conti, Problèmes linéaires pour les équations différentielles ordinaires, Math. Nachr. 23 (1961), 161-168.

[3] G. Conti, R. Iannacci, Using a nonlinear spectral theory to solve boundary value problems, Nonlinear Anal. 6 (1982), 415-421.

[4] M. Grandolfi, Problemi ai limiti per le equazioni differenziali multivoche, Atti Accad. Naz. Lincei, Rend. Classe Sci. Fis. Mat. Nat. 42 (1967), 355-360.

[5] R. I a nnacci, The Spectrum for Nonlinear Multivalued Maps Via Approximations, Boll. Un. Mat. Ital. (5) 15-B (1978).

[6] M. Kisielewicz, Multivalued Differential Equations with Compact Right-Hand Side in Banach Space, preprint.

[7] A. Lasot a, Z. Opial, An Application of the Kakutani-Ky Fan Theorem in the Theory of Ordinary Differential Equations, Bull. Acad. Polon. Sci., Ser. Sci. Math. Astronom. Phys. 13 (1965), 781-786.

[8] L. E. Miller, Generalized Boundary Value Problems, J. Math. Anal. Appl. 74 (1980), 233246.

ISTITUTO DI SCIENZE E TECNOLOGIE, FACOLTȦ DI ARCHITETTURA

UNIVERSITA DI FIRENZE, PIAZZA BRUNELLESCHI, SO121 FIRENZE

DIPARTIMENTO DI MATEMATICA DELLUNIVERSITÁ DELLA CALABRIA

EDIFICIO POLIFUNZIONALE, ARCAVACATA DI RENDE (COSENZA)

Reçu par la Rédaction le 1983.10.15 